\title{
Introduction to the special issue of Space Communication Journal on the ESA Workshop on radiowave propagation - 2011
}

\author{
L. Castanet (Special Issue editor), A. Martellucci and R. Prieto-Cerdeira (Workshop organisers)
}

The last ESA Workshop on radiowave propagation was held from 30th November to 2nd December 2011 in the ESTEC site of the European Space Agency in Noordwijk, The Netherlands. The aim of this workshop was to present recent research and results in the field of propagation modelling, tools and data for Earth-space applications, including Mobile and Fixed Terminal Satellite Communication Systems, Satellite Navigation Systems, Data Downlink for Earth Observation and Space Exploration Systems and for Radio Science experiments. The workshop also promoted coordination and collaboration between European and non-European organisations in support of ITU-R radio regulatory activities and EU RTD/COST Action IC0802 "Propagation tools and data for integrated Telecommunication, Navigation and Earth Observation systems". This workshop was preceded by the 1st European School on Radiowave Propagation for Space Applications, organized by ESA and COST IC0802 in order to give the occasion to students and early stage Researchers to interact and discuss with organizations performing R\&D in this field.

This workshop was considered a success in terms of quality of contributions, attendance, and topics addressed and for this reason, the best papers have been selected to be published in this special issue of Space Communications Journal.

The Workshop incorporated sessions on the following topics:

- Propagation Modelling for Slanth Path Radio Links at Ka Band and above

- Propagation Data, Tools and Models for the Design of SatCom Systems
- Propagation Experiments for Fixed SatCom Systems

- Propagation and Interference Effects in Global Navigation Satellite Systems (GNSS)

- Propagation Channel Models for Mobile Satellite Systems and Mobile Broadcasting

- Propagation Experiments for Mobile SatCom Systems

- Tropospheric Effects on Radio-science Experiments

The selected papers have been carefully chosen and peer reviewed by the following members of the ESA workshop Technical Committee and additional wellknown propagation experts:

- Cesar Amaya, CRC, Canada

- Laurent Castanet, ONERA, France

- Uwe-Carsten Fiebig, DLR, Germany

- Nicolas Floury ESA/ESTEC, The Netherlands

- Fernando Perez Fontan, University of Vigo, Spain

- Antonio Martellucci, ESA/ESTEC, The Netherlands

- Franck Marzano, University of Rome, Italy

- Vinia Mattioli, University of Rome, Italy

- Athanasios Panagopoulos, NTIA, Greece

- Marlene Pontes, CETUC, Brasil

- Roberto Prieto-Cerdeira, ESA/ESTEC, The Netherlands

- Michael Schoenhuber, Joanneum Research, Austria

- Luiz Silva Mello, CETUC, Brasil

- Carlo Riva, Politecnico di Milano, Italy

- Dave Rogers CRC, Canada 
- Mike Willis, STFC-RAL, United Kingdom

- Danielle Vanhoenacker-Janvier, UCL, Belgium

The majority of the papers were considered of high quality for a journal publication with minor modifications, and after a difficult selection, papers covering a diverse list of topics were selected for the journal including tropospheric scintillation, attenuation time-series synthesizers, rain field generators, longterm analysis of propagation experiments, propagation tools for reconfigurable antenna analysis, diversity experiments, channel models in urban environments for Land Mobile Satellite and GNSS including antenna arrays and MIMO and calibration systems for microwave radiometers. 